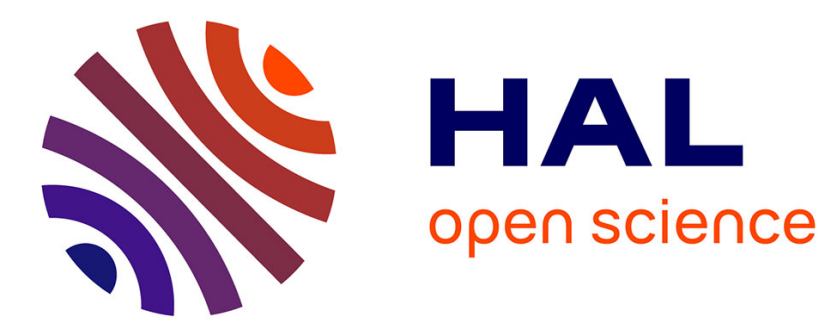

\title{
Evidence of guided acoustic waves propagating along a micrometric fiber
}

\author{
Haithem Khelfa, Denis Mounier, Christophe Poilâne, Pascal Picart
}

\section{To cite this version:}

Haithem Khelfa, Denis Mounier, Christophe Poilâne, Pascal Picart. Evidence of guided acoustic waves propagating along a micrometric fiber. Applied Physics Letters, 2014, 10.1063/1.4899195] . hal-03347180

\author{
HAL Id: hal-03347180 \\ https://hal.science/hal-03347180
}

Submitted on 17 Sep 2021

HAL is a multi-disciplinary open access archive for the deposit and dissemination of scientific research documents, whether they are published or not. The documents may come from teaching and research institutions in France or abroad, or from public or private research centers.
L'archive ouverte pluridisciplinaire $\mathbf{H A L}$, est destinée au dépôt et à la diffusion de documents scientifiques de niveau recherche, publiés ou non, émanant des établissements d'enseignement et de recherche français ou étrangers, des laboratoires publics ou privés. 


\title{
Evidence of guided acoustic waves propagating along a micrometric fiber
}

\author{
Haithem Khelfa, ${ }^{1}$ Denis Mounier, ${ }^{2, a)}$ Christophe Poilâne, ${ }^{3}$ and Pascal Picart ${ }^{1}$ \\ ${ }^{1}$ Université du Maine, LAUM, CNRS UMR 6613, 72085 Le Mans, France \\ ${ }^{2}$ Université du Maine, IMMM, CNRS UMR 6283, 72085 Le Mans, France \\ ${ }^{3}$ Normandie University, 14032 Caen, France; UNICAEN, CIMAP, 14032 Caen, France; ENSICAEN, 14050 \\ Caen, France; CEA, UMR6252, 14070 Caen France; and CNRS UMR6252, 14050 Caen, France
}

(Received 12 September 2014; accepted 10 October 2014; published online 21 October 2014)

\begin{abstract}
We propose a contactless optical method to determine the dispersion curves of guided acoustic modes propagating along a micrometric fiber. Subnanosecond laser pulses are used to generate guided acoustic waves, and an optical probe is used for measuring the ultrasonic displacements at the fiber surface. The test sample is an aluminum fiber of diameter $33 \mu \mathrm{m}$. The comparison between the experimental and theoretical dispersion curves is presented. (C) 2014 AIP Publishing LLC.

[http://dx.doi.org/10.1063/1.4899195]
\end{abstract}

The theoretical studies on acoustic waves guided by hollow and solid cylinders ${ }^{1,2}$ have nowadays important applications in the non-destructive testing ${ }^{3}$ (NDT) and the evaluation of the elastic properties ${ }^{4}$ of pipes. In this letter, we demonstrate the potential application of guided acoustic waves for the evaluation of elastic properties of a single micrometric fiber. The most challenging problem with a fiber is to excite and detect guided acoustic waves and determine their dispersion curves, which will lead to the determination of elastic constants.

The elastic properties of elementary fibers used in reinforced composite materials must be known with the best accuracy in order to predict their mechanical behavior. Elementary fibers have diameters in the range of $5-50 \mu \mathrm{m}$ and at least lengths of several centimeters. For most fibers, the diameter is constant over more than $1 \mathrm{~mm}$ of fiber, so that the infinite cylinder model provides a good approximation for a fiber. Moreover, the elastic properties of most fibers are transversely isotropic. Taking the fiber axis as the isotropy axis and the third axis (z-axis) of a Cartesian coordinate system linked to the fiber, the elastic properties of the fiber material are completely defined by five independent elastic constants: $C_{11}=C_{22}, C_{12}, C_{13}, C_{33}, C_{44}=C_{55}$. The two elastic constants $C_{11}$ and $C_{33}$ determine the velocities of plane longitudinal waves that propagate respectively along the $x$-axis (or the $y$ axis) and the $z$-axis. Such plane acoustic waves can be evidenced only for acoustic wavelengths that are small compared to the fiber diameter $d$, which means frequencies well beyond $1 \mathrm{GHz}$. Nevertheless, such high frequency acoustic waves can be generated using subpicosecond laser pulses. The velocity of acoustic waves traveling back and forth across a fiber diameter can be measured, ${ }^{5}$ leading to the evaluation of the elastic constants $C_{11}$ and $C_{22}$. For small objects such as a single fiber, the use of a contactless technique of measurement is essential. The use of a pump laser, either a continuous wave (CW) laser modulated sinusoidally ${ }^{6}$ or a pulsed laser, is an efficient technique of exciting acoustic waves within a fiber without contact. Another laser beam (the probe beam) is used for the detection of the ultrasonic vibrations. This pump-probe optical

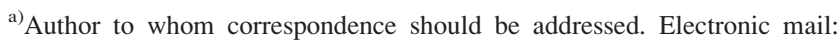
denis.mounier@univ-lemans.fr
}

technique, referred to as laser ultrasonics (LU), has been used for investigating circumferential acoustic waves that propagate on the surface of cylinders, i.e., whispering gallery and Rayleigh waves with wavelengths that are small compared to the cylinder diameter. ${ }^{7-10}$ Alternatively, Laser Resonant Ultrasound Spectroscopy (LRUS) is a variant of LU which focuses on the measurement of eigenfrequencies. Recently, we applied LRUS to a single aluminum fiber by measuring cross-section eigenfrequencies. But this method permits to retrieve only two independent elastic constants: $C_{11}$ and $C_{66}=\left(C_{11}-C_{12}\right) / 2$, for instance. ${ }^{11}$ Thus, for the complete evaluation of the elastic constants of an anisotropic fiber, it is essential to characterize acoustic waves that propagate along the fiber axis. One possible approach is the use of bulk conical or surface helical acoustic waves in the fiber, ${ }^{12}$ which would require frequencies much beyond $1 \mathrm{GHz}$. On the other hand, the study of guided acoustic waves that propagate along an anisotropic fiber is possible below $1 \mathrm{GHz}$ and would permit the evaluation of elastic constants. ${ }^{4}$

An acoustic wave guided by a cylinder (a guided acoustic mode) is characterized both by an axial wave vector $k=2 \pi / \lambda$ and an angular frequency $\omega$. The dispersion relations $\omega(k)$ can be calculated if the elastic properties of the fiber material are known. Cross-section eigenmodes are particular modes for which $k=0$. The dispersion curves which start with a horizontal slope at $k=0$ and for which $\omega(0) \neq 0$ are of particular interest. If $k \approx 0$, the group velocities $v_{g}=d \omega / d k$ of the modes are small compared to the lowest velocity $c_{T}$ of bulk acoustic waves. However, the assumption $v_{g} \ll c_{T}$ is only valid for rather long acoustic wavelengths $\lambda$, typically 2-3 times the fiber diameter $d$.

For the guided modes of a cylinder with a circular crosssection, we use both the nomenclature of Silk and Bainton $(\mathrm{SB})^{13}$ and that of Viktorov ${ }^{8,9,14}$ for circumferential waves (waves for which $k=0$ ). The guided modes are named $X(m, n)$ (or simply $X m, n$ in Figures 1 and 3), where the letter symbols $X=L, W G, R, S$, or $T$ represent, respectively: longitudinal, whispering gallery, Rayleigh, axial shear, and torsional modes. The positive integer $m$ characterizes the rotational symmetry of the mode around the cylinder axis. The positive integer $n$ orders the dispersion curves $\mathrm{Xm}$ according to the increasing frequencies for $k=0$. According to the nomenclature of 


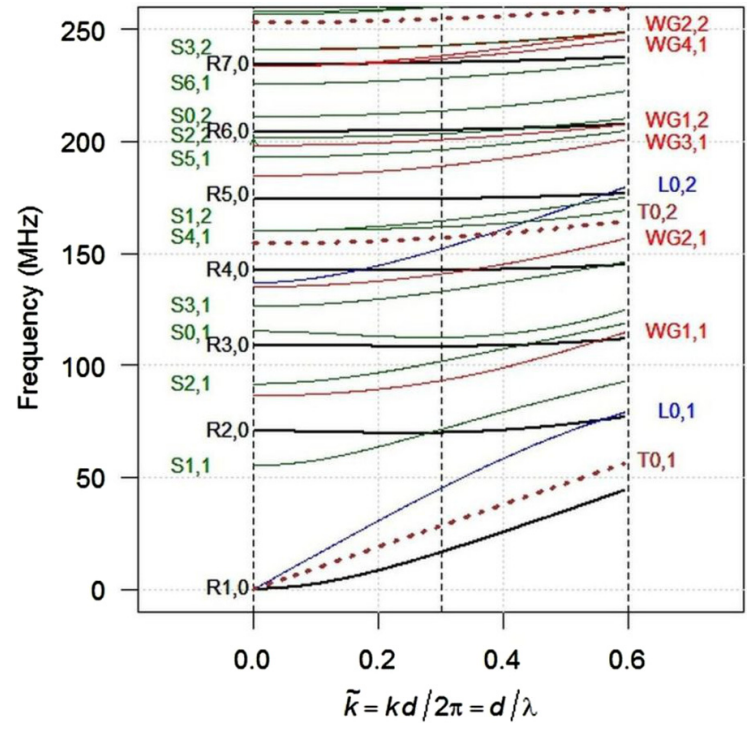

FIG. 1. Dispersion curves of an aluminum fiber calculated with the following parameters: diameter $d=32.7 \mu \mathrm{m}$, Young's modulus $E=69.6 \mathrm{GPa}$, Poisson's ratio $\nu=0.35$ and density $\rho=2700 \mathrm{~kg} / \mathrm{m}^{3}$ (bulk density). Young's modulus and Poisson's ratio are the values obtained by fitting the crosssection eigenfrequencies of Fig. 3.

Viktorov, we split the flexural (F) modes in the SB nomenclature into two separate categories: whispering gallery $(W G)$ and Rayleigh $(R)$ modes.

The displacement field of a guided mode $\mu(=X(m, n))$ is expressed, in cylindrical coordinates $(r, \theta, z)$, as follows:

$$
\mathbf{U}(r, \theta, z, t) \mid \begin{array}{l|l}
U_{r}^{\mu}(r, k) & \begin{array}{l}
\cos (m \theta) \cos (\omega t-k z) \\
\sin (m \theta) \cos (\omega t-k z)
\end{array} \\
U_{\theta}^{\mu}(r, k) & \begin{array}{l}
\sin (m \theta) \cos (\omega t-k z) \\
\cos (m \theta) \cos (\omega t-k z)
\end{array} \\
U_{z}^{\mu}(r, k) & \begin{array}{l}
\cos (m \theta) \sin (\omega t-k z) \\
\sin (m \theta) \sin (\omega t-k z),
\end{array}
\end{array}
$$

which can be written in a compact form

$$
\mathbf{U}(r, \theta, z, t)=\mathbf{U}^{\mu}(r, \theta, k) \exp [i(\omega t-k z)]+c . c .,
$$

where $\mathbf{U}^{\mu}(r, \theta, k)$ is the displacement of a cross-section. The notation c.c. stands for the complex conjugate. There are two-fold degenerate modes for $m \neq 0$ and non-degenerate modes for $m=0$. Longitudinal modes $L(0, n)$, torsional modes $T(0, n)$ and shear axial modes $S(0, n)$ have a full rotational symmetry around the cylinder axis. For any value of $k$, the orthoradial displacements are zero for L-modes, and for T-modes, only the orthoradial displacements are non-zero. Provided $k=0$, the displacements of axial shear modes $S(m, n)$ are exclusively along the $z$-axis. But if $|k|>0, U_{r} \neq 0$ and/or $U_{\theta} \neq 0$ outside the nodal points of vibration. On the contrary, if $k=0$, the axial displacements $U_{z}$ are zero for $W G(m, n)$ modes $(n>0)$ and $R(m, 0)$ modes. For Rayleigh modes $R(m, 0)$, radial displacements near the fiber surface tend to predominate orthoradial displacements, and for the modes $W G(m, 1)$, it is the reverse.

Figure 1 shows the dispersion curves of an aluminum fiber, calculated by solving the secular equations given by Mirsky, ${ }^{2}$ which are applicable for transversely isotropic materials and thus for isotropic aluminum. We consider only modes with real $k$, which can propagate along the fiber, though Zemanek ${ }^{15}$ pointed out the existence of evanescent waves with purely imaginary or complex values of $k$. For a solid cylinder, the dispersion curve of the first Rayleigh mode $R(1,0)$ is similar to that of the antisymmetric Lamb mode $A_{0}$ of a plate. ${ }^{16}$ If $k \neq 0$, the two basic mode shapes of $R(1,0)$ show undulation of the cylinder, either in the $z x$ - or $y z$-plane. The dispersion curve of the $L(0,1)$ mode has a constant slope for $k \approx 0$, with a group or phase velocity: $C_{b}(k \approx 0)=\sqrt{E / \rho}$, where $E$ is Young's modulus and $\rho$ the density. Shear axial modes are interesting to consider as they involve strongly the elastic constants $C_{44}$ and $C_{55}$ for $k \approx 0$.

As the acoustic waves are generated by the sudden heating of a short laser pulse focused onto the fiber surface, ${ }^{11}$ a lot of guided acoustic modes are excited simultaneously. The shape of the pump spot determines the spanning of guided modes that are excited both in the $\omega$ - and $k$-domains. The guided acoustic modes are detected, at a distance $z$ from the pump spot, through the measurement of the radial displacements $U(z, t)=U_{r}$ in function of the time $t$, by a probe beam focused at the point $z$ of the fiber surface. The measured signal $U(z, t)$ resulting from the superposition of many guided modes can be expressed as follows:

$$
U(z, t)=\sum_{\mu=1}^{\infty} \int_{-\infty}^{+\infty} \tilde{U}^{\mu}(k) \exp \left\{i\left[\omega_{\mu}(k) t-k z\right]\right\} d k+c . c
$$

where $\tilde{U}^{\mu}(k)$ represents the vibration amplitude of the mode $\mu$. Each angular frequency is considered as a complex number: $\omega_{\mu}(k)=\omega_{\mu}^{\prime}(k)+i \omega_{\mu}^{\prime \prime}(k)$. The phase and group velocities are respectively: $v_{\mu}^{\varphi}(k)=\omega_{\mu}^{\prime}(k) / k$ and $v_{\mu}^{g}(k)=d \omega_{\mu}^{\prime}(k) / d k$, and the mode damping time is $\tau_{\mu}(k)=1 / \omega_{\mu}^{\prime \prime}(k)$.

We assume that the damping is sufficiently weak, so that $\omega_{\mu}(k)$ may be considered as a real number. Then, Eq. (3) can be approximated by a double Fourier integral

$$
U(z, t) \approx \int_{-\infty}^{+\infty} \int_{-\infty}^{+\infty} \tilde{U}(k, \omega) \exp \{i(\omega t-k z)\} d k d \omega+c . c .,
$$

where the density function $\tilde{U}(k, \omega)$ is approximately the Fourier transform of $U(z, t)$. As the function $\tilde{U}(k, \omega)$ is defined in the real $(k, \omega)$-domain, the dispersion curves $\omega_{\mu}(k)$ can be displayed by plotting the image of $|\tilde{U}(k, \omega)|$.

If $\omega_{\mu}(k)$ is a complex number, the dispersion curves can be determined by using the so-called "matrix-pencil" method (MP). ${ }^{17,18}$ We calculate first the Fourier transform of Eq. (3) with respect to $z$, which leads to

$$
\hat{U}(k, t)=\sum_{\mu=1}^{\infty} \tilde{U}^{\mu}(k) \exp \left[i \omega_{\mu}(k) t\right]+c . c .
$$

Then, the MP method can be applied to retrieve both the complex frequencies $\omega_{\mu}(k)=\omega_{\mu}^{\prime}(k)+i \omega_{\mu}^{\prime \prime}(k)$ and the amplitudes $\tilde{U}^{\mu}(k)$.

The focusing of the pump and probe beams on the fiber is depicted on Fig. 2. The sample is an aluminum fiber mounted on a $1 \mathrm{~cm}$ diameter metal ring. The fiber diameter is $32.7 \pm 0.2 \mu \mathrm{m}$, measured with a scanning electron microscope (SEM). The vibrations of the fiber are excited by using 


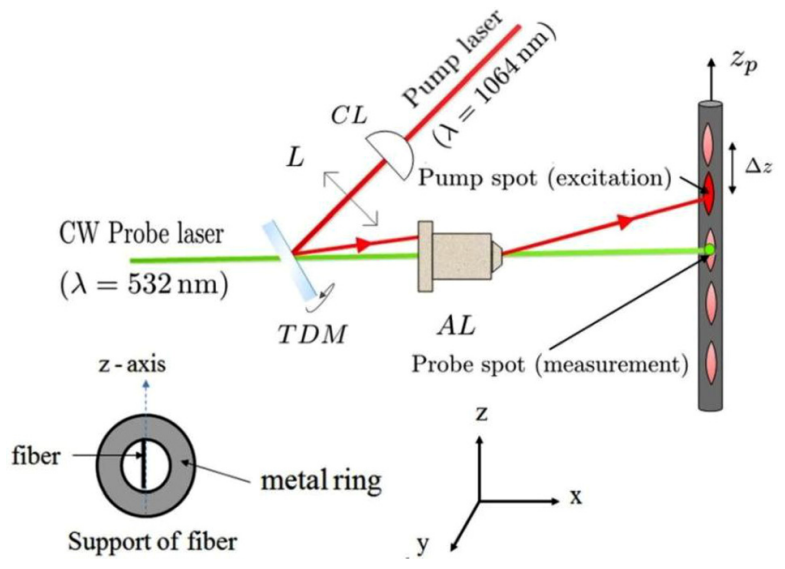

FIG. 2. The laser ultrasonics experimental setup used to study the propagation of guided acoustic waves along a micrometric fiber. $C L$ : cylindrical lens $(f=700 \mathrm{~mm}), L$ : focusing lens, $T D M$ : tilted dichroic mirror, and $A L$ : aspherical lens $(\mathrm{f}=8 \mathrm{~mm}$ and $\mathrm{NA}=0.5)$.

a Q-switched Nd:YAG laser at $1064 \mathrm{~nm}$, which provides $0.6 \mathrm{~ns}$ optical pulses at a repetition rate of $4 \mathrm{kHz}$. A pulse energy of $100 \mathrm{~nJ}$ is used to excite the vibrations of the fiber in the thermoelastic regime. The laser beam of a stabilized homodyne Michelson interferometer, with a wavelength of $532 \mathrm{~nm}$ and a CW power of $2 \mathrm{~mW}$, is focused onto the fiber surface to measure the radial displacements. ${ }^{11}$ Both the pump and probe beams are focused by the aspherical lens (AL). The probe spot has a Gaussian diameter at $1 / e^{2}$ of $2 w_{s}=1.5 \mu \mathrm{m}$ at the fiber surface. By inserting a cylindrical lens (CL) in the optical path of the pump beam, we obtain a Gaussian elliptic spot at the fiber surface with the full width and length at $1 / e^{2}$ of $2 w_{y} \times 2 w_{z}=4 \mu \mathrm{m} \times 72 \mu \mathrm{m}$, the main axis of the ellipse being aligned with the fiber axis. The length $2 w_{z}$ of the pump spot determines the Gaussian distribution of guided modes that are excited in the $k$-domain, which is characterized by the dimensionless standard deviation $\tilde{k}_{c}=k_{c} d / 2 \pi=d / \pi w_{z} \approx 0.3$. Indeed, the last value is $a$ priori chosen to favor the excitation of modes with wavelengths $\lambda \geq 3 d$, which have low group velocities.

The pump-probe distance $z$ is changed by moving the pump spot along the fiber axis while the probe spot and the fiber remain fixed. The dichroic mirror (TDM) reflecting the pump beam can be tilted to move the pump spot over $\pm 500 \mu \mathrm{m}$ on both sides of the probe spot (Fig. 2). For each position $z_{i}$ of the pump spot, the vibration signal is recorded during $5 \mu$ s by a $3 \mathrm{GHz}$-bandpass digital oscilloscope, with the sampling frequency of $2 \mathrm{GHz}$. Frequencies beyond $1 \mathrm{GHz}$ are filtered by the $1 \mathrm{GHz}$-bandpass photoreceiver. The vibration temporal signals $U\left(z_{i}, t\right)$ are recorded with a sampling pitch $\Delta z \approx 50 \mu \mathrm{m}$, which determines the dimensionless cutoff frequency $\tilde{k}_{\text {max }}=k_{\text {max }} d / 2 \pi=d / 2 \Delta z \simeq 0.3$, whereas the spectral resolution, determined by the spatial sampling window of $1 \mathrm{~mm}$, is $\Delta \tilde{k} \simeq 0.03$. The fiber boundaries are distant of a few millimeters from the test zone so that no echoes from the fiber boundaries are expected in the signal $U(z, t)$ during the acquisition time because of the weakness of group velocities.

Figure 3 shows the grayscale image of the function $|\tilde{U}(\tilde{k}, \omega / 2 \pi)|$ calculated from the data. The pixel dimensions

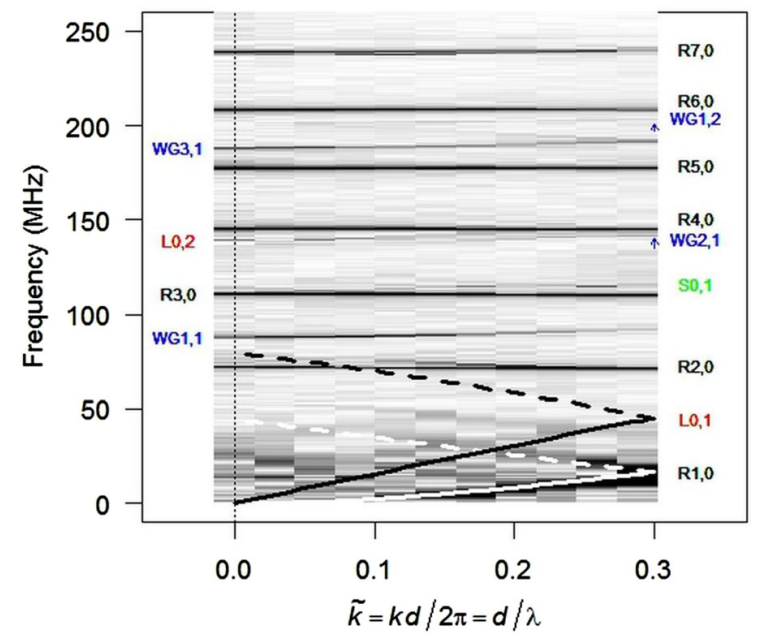

FIG. 3. Image in gray-scale of $|\tilde{U}(k, \omega)|$ revealing the dispersion curves of an aluminum fiber sample of diameter $d=32.7 \mu \mathrm{m}$. The calculated dispersion curves are superimposed for modes $R(1,0)$ (white line) and $L(0,1)$ (black line). The dashed lines are the aliasing part of dispersion curves.

are determined by the spatio-temporal sampling of $U(z, t)$. A white pixel represents a zero amplitude so that the dispersion curves are displayed as gray or dark lines on a white background. The comparison between Fig. 1 and Fig. 3 shows clearly that Rayleigh guided modes are the most easily detected. The whispering gallery modes are less visible than Rayleigh modes. The sub-sampling of $U(z, t)$ in the $z$-domain induces a spectral aliasing in the $k$-domain for the modes: $R(1,0), L(0,1), R(2,0)$ and $W G(3,1)$. Thus, these modes are excited beyond $\tilde{k}=0.3$.

The shear axial mode $S(0,1)$ is only visible for $\tilde{k}>0.2$, which is expected, as radial displacements become significant only for sufficiently large values of $\tilde{k}$. The $R(1,0)$ mode is visible for $\tilde{k}>0.15$ in Fig. 3 , which shows a fairly good agreement between the calculated and experimental dispersion curves.

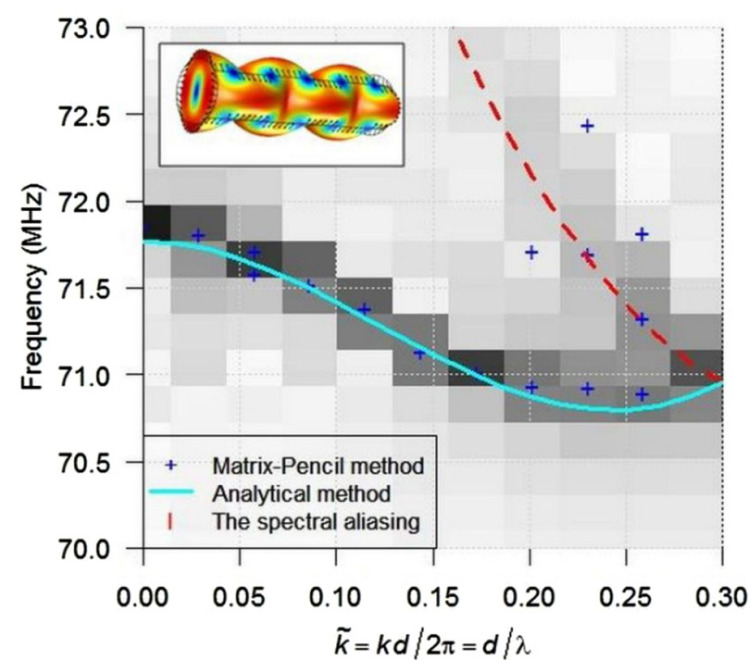

FIG. 4. Calculated dispersion curves (solid line) for the Rayleigh mode $R(2,0)$ superimposed to the image of $|\tilde{U}(k, \omega)|$. The crosses (in blue) represents the experimental frequencies calculated by the "matrix pencil" method. The aliasing part of the calculated dispersion curve is in dashed line (red). Mode shape of $R(2,0)$ for $\tilde{k} \approx 0.4$ (inset). 


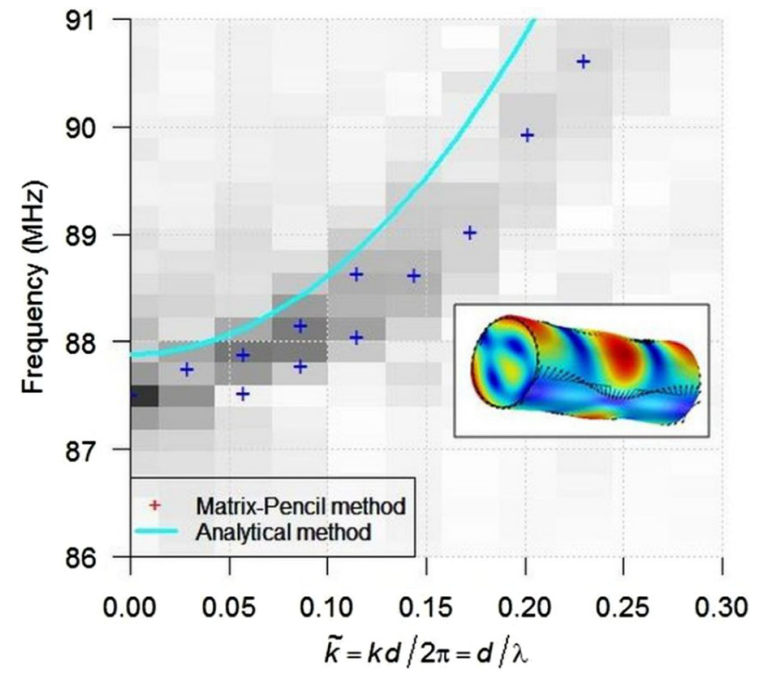

FIG. 5. Calculated and experimental dispersion curves for the mode $W G(1,1)$ with its mode shape for $\tilde{k} \approx 1 / 3$ (inset).

Figure 4 shows the calculated and experimental dispersion curve of the $R(2,0)$ mode, including the aliasing part of the spectrum (dashed curve). The frequencies calculated by the MP method are close to the theoretical frequencies within $0.1 \mathrm{MHz}$ for $\tilde{k}<0.3$ (a relative difference of $0.1 \%$ ). One advantage of the MP method is that the precision in frequency overcomes that given by the Fast Fourier Transform (FFT). The second advantage of the MP method is to permit the evaluation of the damping time of any mode. For the mode $R(2,0)$, we calculate the average damping time $\tau_{\mu}(k) \approx 2 \mu$ s for $\tilde{k}<0.3$.

Figure 5 shows the comparison between the experimental and theoretical dispersion curves, for the first whispering gallery mode $W G(1,1)$. The experimental frequencies are lower than the calculated frequencies by about $0.6 \%$. The MP method reveals a doublet of frequencies for some values of $k$, which is due to the degeneracy lifting induced by the small ellipticity of the fiber cross-section. Finite element modeling confirms that a very small ellipticity of the crosssection induces a significant splitting of the $W G(1,1)$ doublet, whereas a negligible splitting exists for Rayleigh modes and some other WG-modes.

The fairly good agreement between the predicted and experimental dispersion curves demonstrates the propagation of guided acoustic waves along the isotropic aluminum fiber. In the near future, this technique will be applied to determine experimentally the dispersion curves of elementary fibers used in composite materials with the aim of determining their elastic constants.

Haithem Khelfa is grateful to Campus France for the financial support provided by the Eiffel excellence scholarship program 2014.

${ }^{1}$ D. C. Gazis, J. Acoust. Soc. Am. 31, 568 (1959).

${ }^{2}$ I. Mirsky, J. Acoust. Soc. Am. 37, 1016 (1964).

${ }^{3}$ M. Lowe, D. Alleyne, and P. Cawley, Ultrasonics 36, 147 (1998).

${ }^{4}$ D. Gsell and J. Dual, Ultrasonics 43, 123 (2004).

${ }^{5}$ D. Ségur, Y. Guillet, and B. Audoin, Appl. Phys. Lett. 97, 031901 (2010).

${ }^{6}$ C. Prada, O. Balogun, and T. W. Murray, Appl. Phys. Lett. 87, 194109 (2005).

${ }^{7}$ D. Royer, E. Dieulesaint, X. Jia, and Y. Shui, Appl. Phys. Lett. 52, 706 (1988).

${ }^{8}$ B. Audoin, Y. Pan, C. Rossignol, and N. Chigarev, Ultrasonics 44, e1195 (2006).

${ }^{9}$ D. Clorennec, D. Royer, and H. Walaszek, Ultrasonics 40, 783 (2002).

${ }^{10}$ Y. Pan, C. Rossignol, and B. Audoin, J. Acoust. Soc. Am. 115, 1537 (2004).

${ }^{11}$ D. Mounier, C. Poilâne, H. Khelfa, and P. Picart, Ultrasonics 54, 259 (2014).

${ }^{12}$ M. Perton, B. Audoin, Y. D. Pan, and C. Rossignol, J. Acoust. Soc. Am. 119, 3752 (2006).

${ }^{13}$ M. Silk and K. Bainton, Ultrasonics 17, 11 (1979).

${ }^{14}$ I. Viktorov, Rayleigh and Lamb Waves (Plenum Press, New York, 1967).

${ }^{15}$ J. Zemanek, J. Acoust. Soc. Am. 51, 265 (1972).

${ }^{16}$ Z. Su, L. Ye, and Y. Lu, J. Sound. Vib. 295, 753 (2006)

${ }^{17}$ T. K. Sarkar and O. Pereira, IEEE Antennas Propag. Mag. 37, 48 (1995).

${ }^{18}$ F. Schöpfer, F. Binder, A. Wöstehoff, T. Schuster, S. von Ende, S. Föll, and R. Lammering, CEAS Aeronaut. J. 4, 61 (2013). 